\title{
Smartphone Fundoscopy
}

Dear Editor:

More ophthalmologists than ever carry a smartphone with them on a daily basis.

There are many ophthalmic applications, “apps”, and reference texts available on a variety of platforms.

The potential for retinal imaging has been discussed previously in this journal by Lord et al $^{1}$ This included capturing slit lamp images by holding the phones camera to an eye piece, similarly it can be held to an operating microscope. This group also described using indirect ophthalmoscopy by holding a 20D indirect lens in one hand and a pen torch with the smartphone in the other. This author found this technique to be cumbersome and difficult to capture good quality images. Directing a pen torch light and allowing the camera to focus, as well as needing to press the touchscreen to take an image is not user friendly and is unlikely to be used by many ophthalmologists.

There are a number of newer models of available smartphones which have a 5megapixel or greater forward facing camera with inbuilt flash and video capabilities.

I describe here an easy to use method for capturing good quality fundus images.

On video mode set the flash to "on" so it is constantly illuminated. This acts as coaxial powerful light source for the lens of the camera. The ophthalmologist holds the 20D or 28D lens as they would normally when performing indirect ophthalmoscopy and has the smartphone in the other. It is then possible to maintain 
full control of the smartphone in one hand including the ability to focus and magnify the image as desired. Once recording is completed a still image can then be captured from the video sequence and saved or shared as necessary. See figures 1, 2 and 3 available at http://aaojournal.org

The principles of information security require that all reasonable care is taken to prevent inappropriate access, modification or manipulation of data from taking place. In the case of the National Health Service (NHS), the most sensitive of our data is patient record information.

Information governance policies are in place to ensure these principles are upheld by setting clear guidelines for all NHS users. ${ }^{2}$ It is expected that users shall maintain the confidentiality of personal data entrusted to them in accordance with the Data Protection Act 1998 and Access to Health Records Act 1990. The NHS Mail Email service allows for secure patient information to be shared amongst healthcare workers within the $\mathrm{NHS}^{3}$. Similar policies almost certainly exist in other countries.

As the ease of imaging and sharing of diagnostic images increases, there is a corresponding increased potential for specialist input from a distance. Less experienced ophthalmologists may find this technique of imaging very useful for getting advice from senior colleagues, particularly in on-call situations. There is also potential for it's use in resource-poor settings

Andrew Bastawrous, MRCOphth, Liverpool, UK. 
Figure legends:

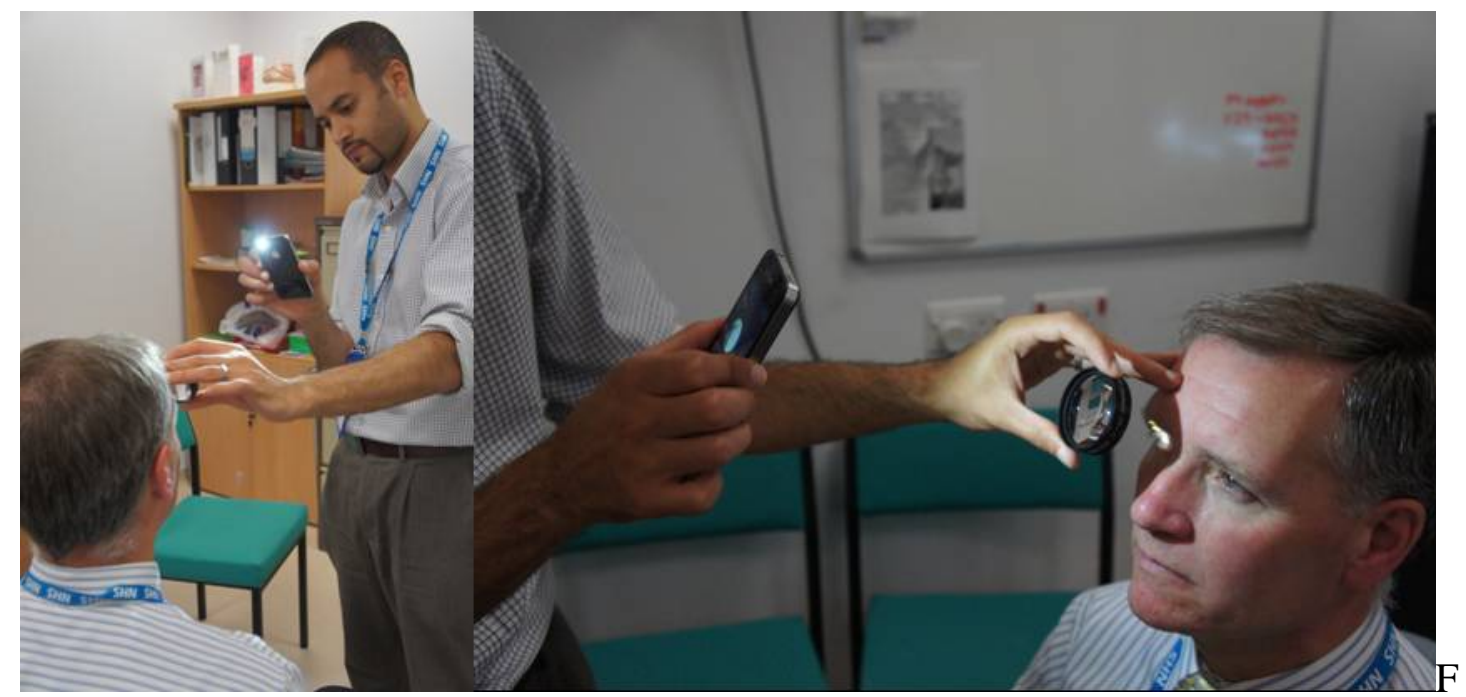

Figure 1a \& 1b. Smartphone with 20D Indirect lens used to capture a fundus image

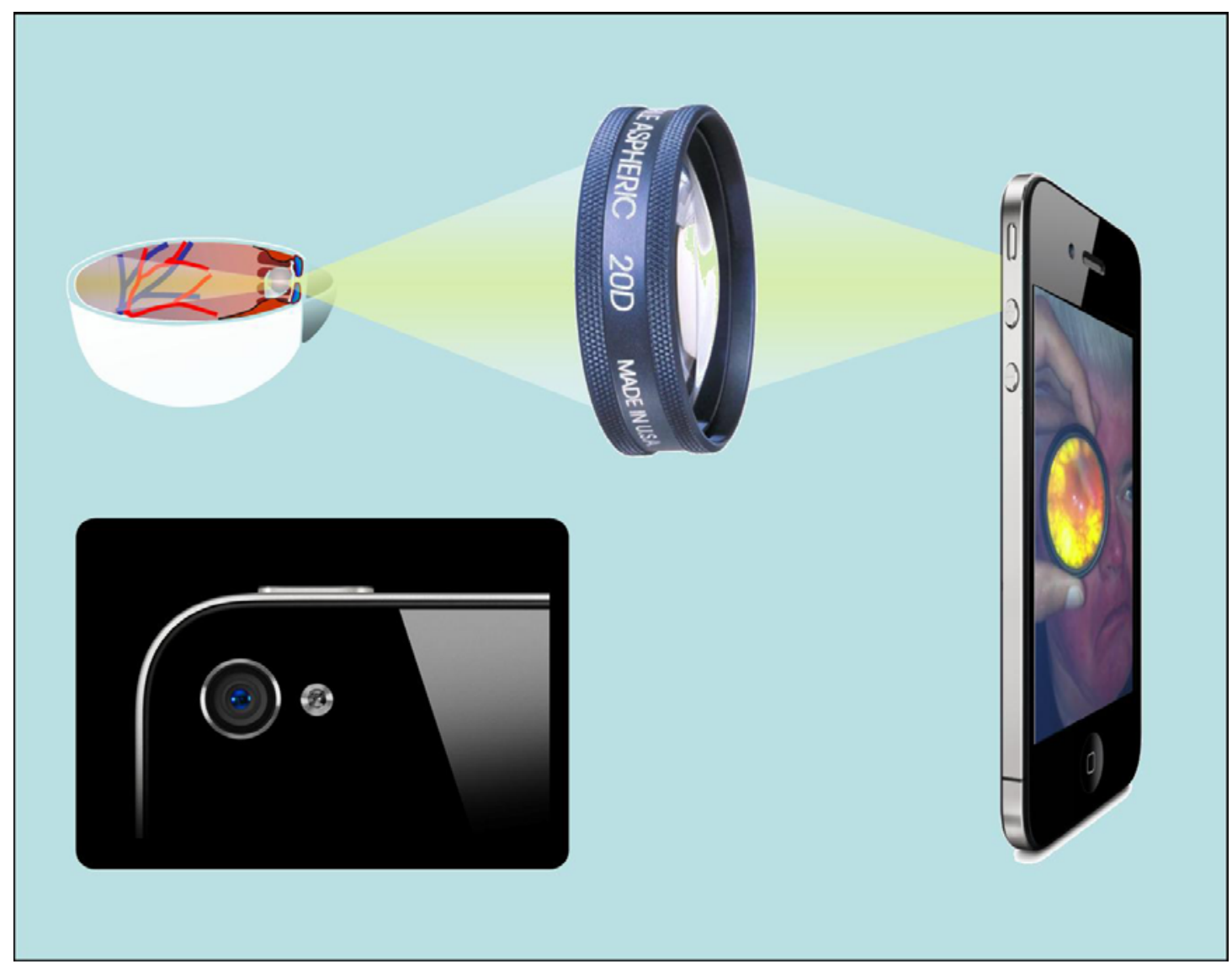

Figure 2. Animation depicting the use of a smartphone with a 20D Indirect lens 


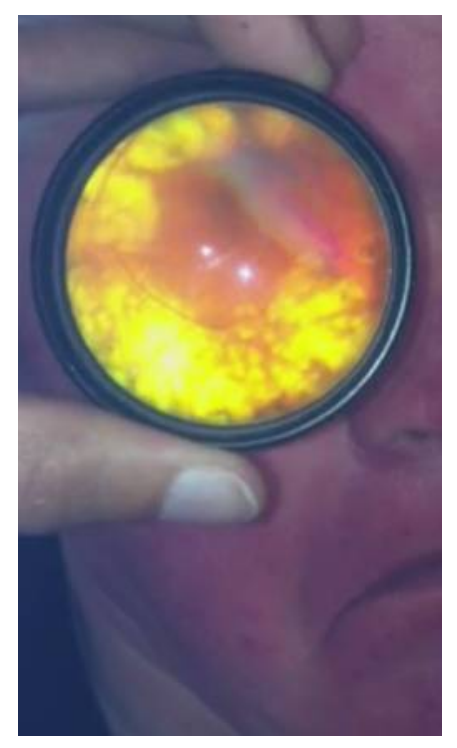

Figure 3. Image of a fundus using the described technique

\footnotetext{
${ }^{1}$ Lord RK, Shah VA, San Filippo AN, Krishna R. Novel Uses of Smartphones in Ophthalmology. Ophthalmology. 2010 Jun;117(6):1274-1274.e3

${ }^{2}$ Retrieved from: http://www.connectingforhealth.nhs.uk/systemsandservices/infogov/security on 19.07.2011

${ }^{3}$ Retrieved from: http://www.connectingforhealth.nhs.uk/resources/systserv/nhsmail on 19.07.2011
} 